\title{
Cloning and overexpression of PeWRKY 31 from Populus $x$ euramericana enhances salt and biological tolerance in transgenic Nicotiana
}

Xiaoyue Yu ${ }^{1,2}$, Yu Pan ${ }^{1,3}$, Yan Dong ${ }^{1,2}$, Bin Lu ${ }^{4}$, Chao Zhang ${ }^{1,2}$, Minsheng Yang ${ }^{1,2^{*}}$ (D) and Lihui Zuo ${ }^{1,5^{*}}$

\begin{abstract}
Background: As important forest tree species, biological stress and soil salinization are important factors that restrict the growth of Populus $\times$ euramericana. WRKYs are important transcription factors in plants that can regulate plant responses to biotic and abiotic stresses. In this study, PeWRKY31 was isolated from Populus $\times$ euramericana, and its bioinformation, salt resistance and insect resistance were analyzed. This study aims to provide guidance for producing salt-resistant and insect-resistant poplars.

Results: PeWRKY31 has a predicted open reading frame (ORF) of 1842 bp that encodes 613 amino acids. The predicted protein is the unstable, acidic, and hydrophilic protein with a molecular weight of $66.34 \mathrm{kDa}$, and it has numerous potential phosphorylation sites, chiefly on serines and threonines. PeWRKY31 is a zinc-finger $\mathrm{C}_{2} \mathrm{H}_{2}$ type-ll WRKY TF that is closely related to WRKY TFs of Populus tomentosa, and localizes to the nucleus. A PeWRKY31 overexpression vector was constructed and transformed into Nicotiana tabacum L. Overexpression of PeWRKY31 improved the salt tolerance and insect resistance of the transgenic tobacco. Transcriptome sequencing and KEGG enrichment analysis showed the elevated expression of genes related to glutathione metabolism, plant hormone signal transduction, and MAPK signaling pathways, the functions of which were important in plant salt tolerance and insect resistance in the overexpressing tobacco line.
\end{abstract}

Conclusions: PeWRKY31 was isolated from Populus $\times$ euramericana. Overexpression of PeWRKY31 improved the resistance of transgenic plant to salt stress and pest stress. The study provides references for the generation of stress-resistant lines with potentially great economic benefit.

Keywords: WRKY, Genetic transformation, Salt tolerance, Insect resistance, Transcriptome

\footnotetext{
*Correspondence: yangms100@126.com; zuolihui001@163.com

'Forest Department, Forestry College, Hebei Agricultural University, Baoding, China

Full list of author information is available at the end of the article
}

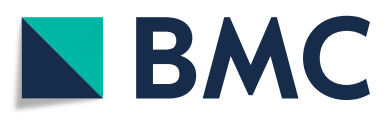

(C) The Author(s). 2021 Open Access This article is licensed under a Creative Commons Attribution 4.0 International License, which permits use, sharing, adaptation, distribution and reproduction in any medium or format, as long as you give appropriate credit to the original author(s) and the source, provide a link to the Creative Commons licence, and indicate if changes were made. The images or other third party material in this article are included in the article's Creative Commons licence, unless indicated otherwise in a credit line to the material. If material is not included in the article's Creative Commons licence and your intended use is not permitted by statutory regulation or exceeds the permitted use, you will need to obtain permission directly from the copyright holder. To view a copy of this licence, visit http://creativecommons.org/licenses/by/4.0/ The Creative Commons Public Domain Dedication waiver (http://creativecommons.org/publicdomain/zero/1.0/) applies to the data made available in this article, unless otherwise stated in a credit line to the data. 


\section{Background}

A wide variety of plant transcription factors interact with defense-related gene promoters, ultimately enhancing plant resistance to stress [1]. The transcriptional regulation of stress response genes not only helps plants respond to various stressors [2], but is also important for plant growth, development, and metabolic pathways [3]. Transcription factors, present in various families with complex functions, play an important role in plant development, metabolism, and responses to biotic and abiotic stresses. Transcription factors such as NAC, AP2/ ERF, and WRKY play important roles in the regulation of plant growth under stress [4].

WRKYs form one of the largest transcription factor families, with the seventh-highest numbers among the higher plant transcription factor families, behind bHLH, MYB, ERF, NAC, $\mathrm{C}_{2} \mathrm{H}_{2}$, and bZIP [5]. WRKYs have been identified in 165 plants, a total of 14,549 of which are predicted to be involved in responses to biological and abiotic stresses, the transmission of hormone signals, and the regulation of growth and development $[6,7]$. In abiotic stress, WRKYs mainly function in stresses caused by cold and heat [8], drought and flood [9-11], high salt [12-16], and excess radiation [17]. WRKYs also play crucial roles in plant immune responses to biological stress, defense responses to pathogens and bacteria [1820], and in establishing relevant signal transduction pathways [21, 22]. The WRKYs of Arabidopsis and rice have been shown to play a role in insect resistance $[23$, 24], but few studies have investigated poplar WRKYs in the context of insect resistance.

Poplar species are commonly used as model woody plants due to their rapid growth and reproduction, wide distribution, and high adaptability. Additionally, poplar species are commercially important in China. Among them, Populus $\times$ euramericana breeds easily, grows fast and has strong adaptability. In recent years, with the completion of the sequencing of the Populus trichocarpa genome and the continuous development of biotechnology tools, poplar research has been more focused on the molecular level. The latest release of the PlantTFDB (http://planttfdb.cbi.pku.edu.cn/)transcription factor database contains a total of 185 Populus tomentosa WRKYs. Over a dozen related transcription factors have been isolated, their functions have been verified [25], and the WRKY family genes in many poplar trees have been shown to respond to abiotic stress and play important roles in salt resistance [26-28]. Preliminary laboratory research revealed that PeWRKY31 of Populus $\times$ euramericana responded to biological stress, but the underlying mechanism was not clear. In order to clarify the function, PeWRKY31 was isolated from Populus $\times$ euramericana and its sequence was analyzed using bioinformatics in this study. We constructed a vector for subcellular localization analysis and a vector for tobacco overexpression. Then, we identified the role of PeWRKY31 in signal transduction pathways responding to salt and pest stress. The research lays a foundation for the generation of stress-resistant Populus $\times$ euramericana lines.

\section{Results}

\section{Screening and cloning of PeWRKY31}

Total RNA was extracted from leaves of Populus $\times$ euramericana clones and lines that had been treated with $4 \%$ salt or chewed by Clostera anachoreta. The $\mathrm{OD}_{260} /$ $\mathrm{OD}_{280}$ was between 1.8 and 2.0 , and the $\mathrm{OD}_{260} / \mathrm{OD}_{230}$ was greater than 2.0, indicating that the total RNA was of sufficient purity. cDNA was used as a template for determination of PeWRKY31 expression and PCR with the primer set 31\#all $\mathrm{F} / \mathrm{R}$ (Table S1). The target gene PeWRKY31 was amplified by PCR using 31\# All F/R as primer (Table S1). The resulting PCR product was about $1800 \mathrm{bp}$, consistent with the expected size, and was used for carrier construction.

To analyze the expression of PeWRKY31 in Populus $\times$ euramericana under different stress, Populus $\times$ euramericana was treated with $4 \%$ salt and larval feeding. The RT-PCR results showed that the expression of PeWRKY31 changed after treatment with $4 \% \mathrm{NaCl}$ for 7 days and larval feeding for $1 \mathrm{~h}$. The relative expression of PeWRKY31 increased by about 10 times and 8 times, respectively, after $4 \% \mathrm{NaCl}$ treatment and biological stress (Fig. 1), indicating that biological and abiotic stress regulated PeWRKY31 expression.

\section{Bioinformatics analysis of PeWRKY31}

The full-length predicted ORF of PeWRKY31 comprises $1842 \mathrm{bp}$ that encodes 613 amino acids. The predicted

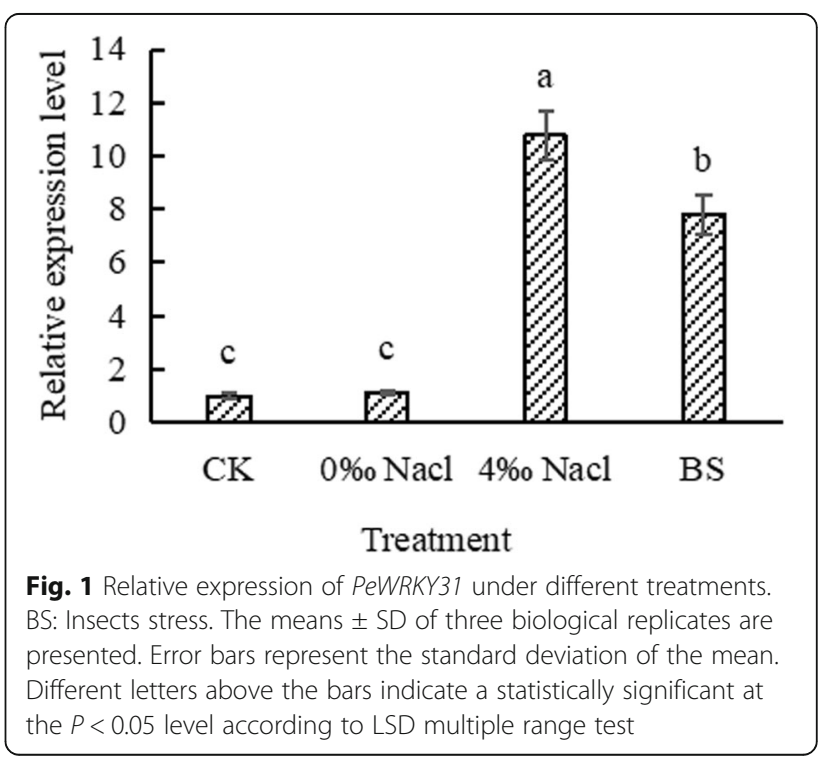


protein has a molecular mass of $66.34 \mathrm{kDa}$, an isoelectric point of 6.12 (acidic), an instability coefficient of 48.59, and a hydrophobicity of -0.712 . These findings indicate that PeWRKY31 is an acidic and unstable hydrophilic protein. In the secondary structure of PeWRKY31, $\alpha-$ helix accounted for $24.96 \%$, $\beta$-rotation for $2.94 \%$, extension chain for $12.56 \%$, and random structures for $59.54 \%$ (Fig. S1-A). The tertiary structure model was mainly composed of five $\beta$-fold structures and random structures (Fig. S1-B). cNLS analysis showed that PeWRKY31 contained the nuclear localization signal sequence PSDN RRR, suggesting that the protein was located on or in the nucleus. Since no predicted transmembrane region was found and there was no predicted signal peptid, the protein is likely intracellular (Fig. S2-A). The protein had a total of 75 potential phosphorylation sites, dominated by serine and followed by threonine.
The amino acid sequences were most closely related to PeWRKY31 in Nicotiana attenuata, Arabidopsis thaliana, Populus trichocarpa, Oryza sativa, Zea mays, and Amygdalus persica were compared using BlastX (https://blast. ncbi.nlm.nih.gov/Blast.cgi), Phytozome v12.1 (https:// phytozome.jgi.doe.gov/pz/portal.html), and DNAMAN (https://www.lynnon.com/dnaman.html) software (Fig. 2a). All seven proteins contained a WRKYGQK sequence and a $\mathrm{C}_{2} \mathrm{H}_{2}$ zinc-finger structure, which were the typical characteristics of Group II WRKY proteins, indicating that they belonged to the class-II WRKY subfamily. PeWRKY31 showed high similarity in WRKY domain compared with WRKY proteins of other species.

Searches were conducted in the NCBI and Phytozome databases (v12.1) using sequences of the PeWRKY31 gene and 15 homologs from seven species (Oryza sativa, Zea mays, Nicotiana attenuata, Amygdalus persica,

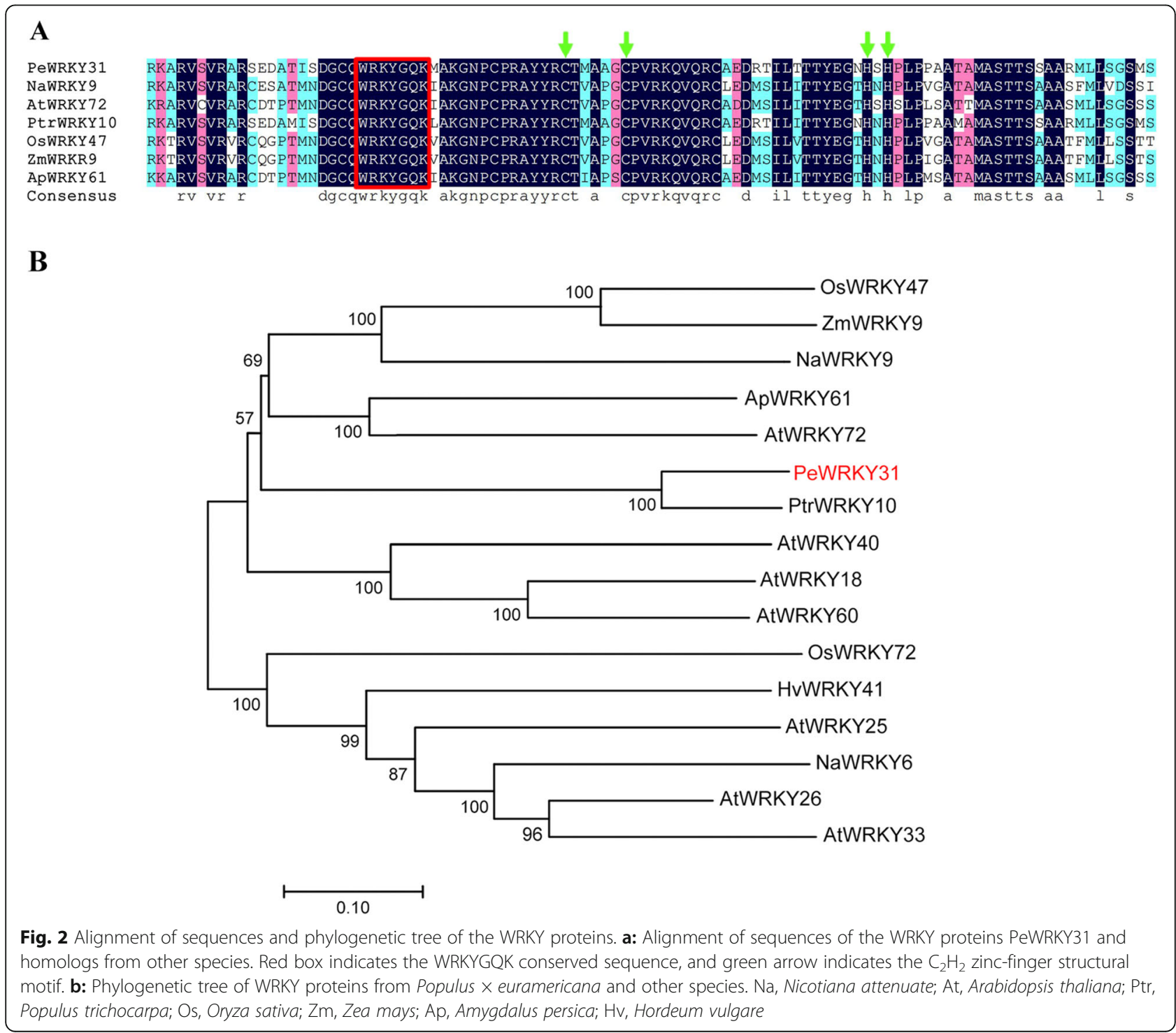


Arabidopsis thaliana, Populus trichocarpa, and Hordeum vulgare). The evolution of genes from different species was analyzed using the neighbor-joining method in MEGA ver. 7.0 (https://www.megasoftware.net) [25], and a phylogenetic tree was constructed using the results (Fig. 2b). PtrWRKY10 had the closest genetic relationship to PeWRKY31, followed by AtWRKY72. The function of PtrWRKY10 was unkonwn, but its closely related AtWRKY72 responded to abiotic stresses such as salt injury [29]. Thus, PeWRKY31 may also respond to salt stress.

\section{Subcellular localization of GFP-PeWRKY31 in transiently transformed tobacco}

The fusion expression vector 35S:GFP-PeWRKY31 (p31\#subl+ 1302) and the control vector 35S:GFP were transiently transformed into tobacco leaves using the corresponding Agrobacterium tumefaciens strains by bacterial infection. The leaves were then observed using a fluorescence microscope (Fig. 3). DAPI fluorescent dye used as a control revealed the locations of nuclei. In leaves transformed with the 35S:GFP vector, the green fluorescent GFP signal was distributed in the nucleus and cytoplasm, indicating non-specific localization, as expected. In leaves transformed with 35S:GFP-PeWRKY31, the GFP fluorescence signal appeared inside cells. Only nuclei showed green fluorescence; no fluorescence signal was detected in the cytoplasm outside nuclei, indicating that GFPPeWRKY31 localized specifically in the nuclei of tobacco leaves. These results show that PeWRKY31 is a nuclearlocalized protein.

\section{Generation of transgenic tobacco lines}

The overexpression vector p31\#op+121(Fig. S3B) was transformed into tobacco using the Agrobacterium tumefaciens-mediated leaf disk method. After $36 \mathrm{~h}$ of coculture followed by screening for resistant vegetative propagules, green resistant vegetative propagules were transferred into rooting medium. Rooted tobacco plantlets were transferred into pots and allowed to grow under normal greenhouse conditions (Fig. 4a-d).

The DNA of ten tobacco lines was subjected to PCR using the specific primers $103 \# \mathrm{~F} / \mathrm{R}$, which amplified an nptII fragment of approximately $500 \mathrm{bp}$ (Fig. 4e), and 35\#send $\mathrm{F}$ and 31\#-JC R, which amplified a $\sim 400$-bp portion of PeWRKY31(Fig. 4f) (Table S1 for all primer information). The plasmid containing the target gene was used as a positive control $(\mathrm{CK}+)$ and WT tobacco was used as a negative control (CK-). The target fragments were amplified from nine of the ten tobacco lines (except lane 1) and the positive control, but not the negative control. Thus, nine of the ten tested tobacco lines were confirmed to contain the target construct.

The qRT-RCR results showed that the relative expression of PeWRKY31 in transgenic lines B5, B12, and A12 was significantly higher than that in the WT, by 10,31 and 17 times, respectively (Fig. 5a). The relative expression of the endogenous homologous gene NtWRKY31

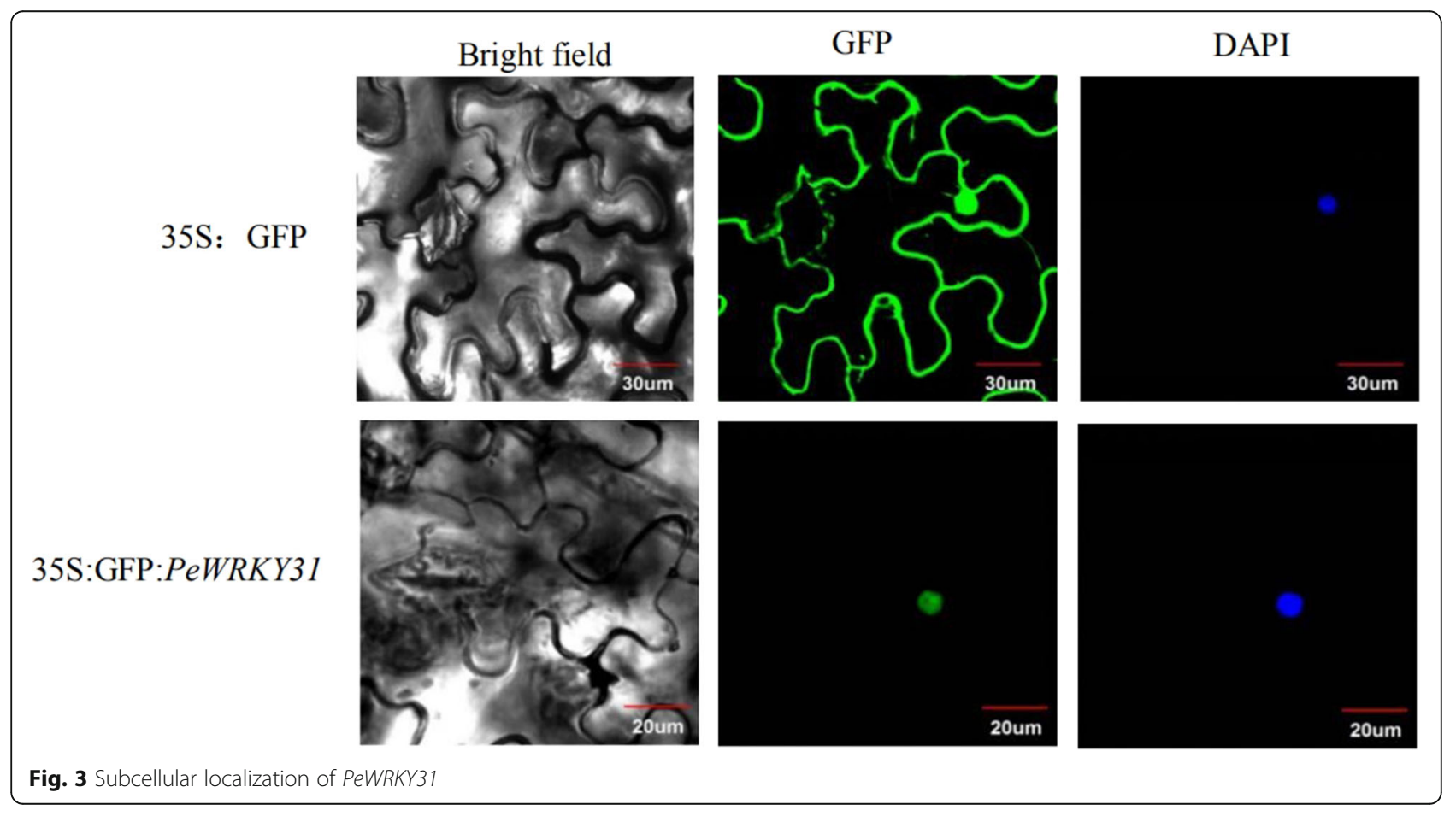


A

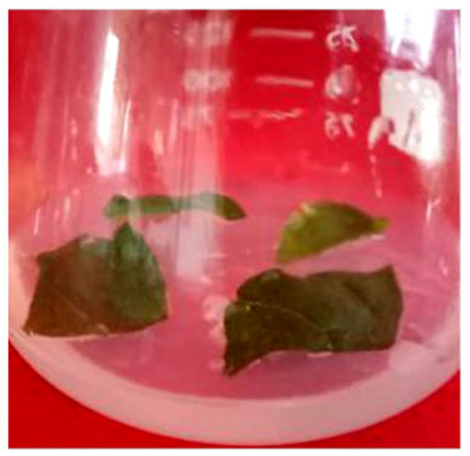

$\mathbf{C}$

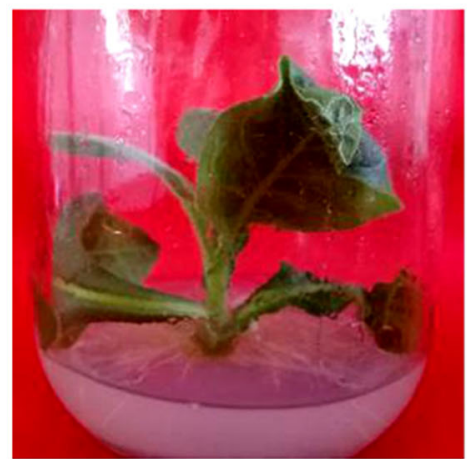

B

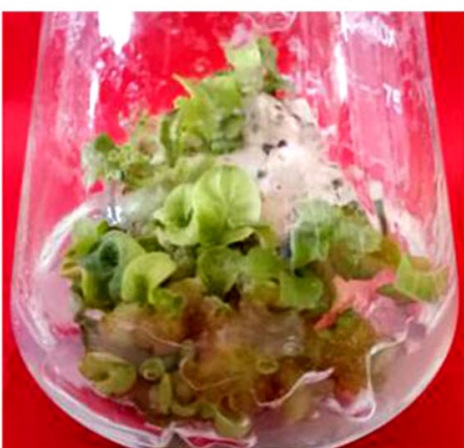

D

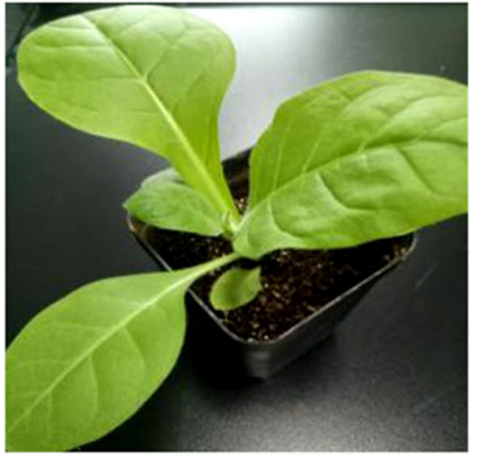

$\mathbf{E}$
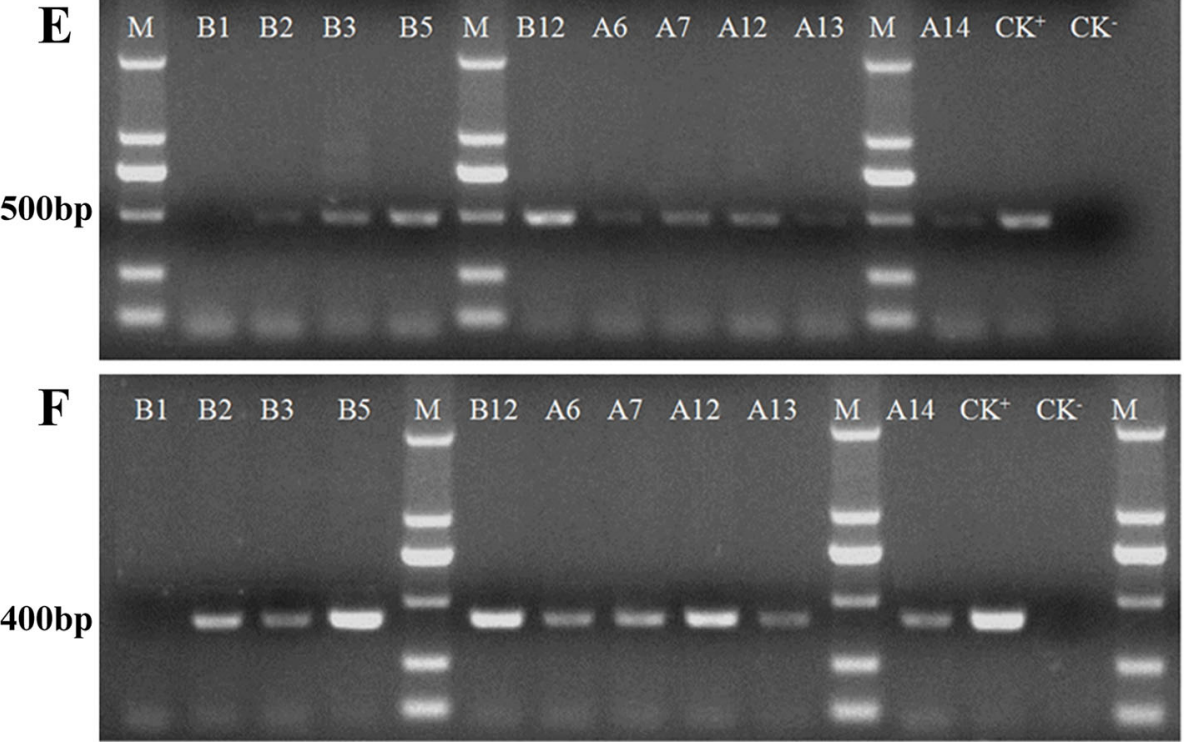

Fig. 4 Generation of PeWRKY31-overexpressing transgenic tobacco and PCR analysis of the ten transformed tobacco lines. a: Leaves used for Agrobacterium infection. b: Screening for resistant vegetative propagules. c: Rooted plantlet. d: Transplanted plantlet. e: $n p t / l$ gene fragment ( $500 \mathrm{bp}$ ) amplified from transgenic tobacco. f: Portion of PeWRKY31 gene ( $400 \mathrm{bp})$ amplified from transgenic tobacco. Lane M, DL2000 DNA marker of US EVERBRIGHT; other lanes, test samples

(LOC107770788) was significantly lower in transgenic lines B5 and A12 than in the WT (Fig. 5b).

\section{Salt tolerance analysis of transgenic tobacco overexpressing PeWRKY31}

The activities of the antioxidant enzymes SOD and POD in plants reflect the degree of stress damage to plants. The SOD and POD activities in the WT and transgenic tobacco lines were lower after $72 \mathrm{~h}$ of $0 \%$ salt stress (Fig. 6). In the presence of a $4 \%$ salt solution, the SOD and POD activities were increased in all lines wheras were significantly higher in the transgenic lines than in WT plants.

The MDA content and electrical conductivity reflect the integrity of the cell membrane. After $72 \mathrm{~h}$ of salt stress, there was no difference in the MDA content or 

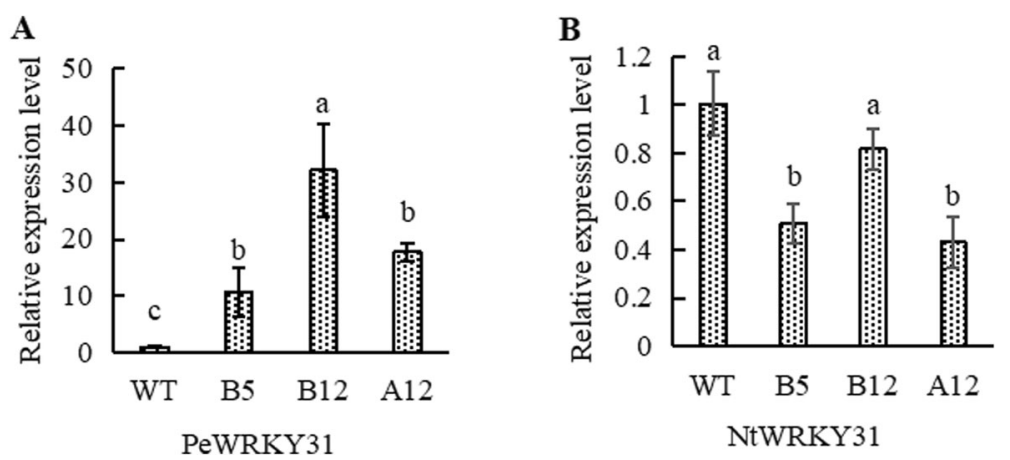

Fig. 5 Relative expression level of qRT-PCR. a: Relative expression of PeWRKY31 in different transgenic lines. b: Relative expression of NtWRKY31 in different transgenic lines. The means \pm SD of three biological replicates are presented. Error bars represent the standard deviation of the mean. Different letters above the bars indicate a statistically significant at the $P<0.05$ level according to LSD multiple range test

the relative conductivity in WT tobacco and the transgenic lines under $0 \%$ salt concentration. In the presence of $4 \%$ salt, the MDA content of each line increased significantly, and the relative conductivity increased from $40 \%$ to $60-80 \%$ compared to the treatment without salt stress. The MDA content and relative conductivity of the transgenic lines were significantly lower than those of the WT, but there was no difference in the results among the transgenic lines. These results showed that salt stress caused oxidative damage to the cell membranes of all of the tobacco lines, and the cell membrane damage in the WT was more serious than in any of the transgenic strains. Therefore, the target gene had a protective effect on the cytoplasmic membranes and improved the salt tolerance of tobacco.

The plant height and ground diameter of WT tobacco and the transgenic lines were monitored throughout the 5 -day period (Fig. 6). The plant height growth rates of all tobacco lines decreased significantly under salt stress; the growth in height and the ground diameter growth of the transgenic lines, especially lines B12, were significantly higher than that of the WT, indicating that the transgenic lines had more robust growth than the WT under salt stress. This indicated that the overexpression of PeWRKY31 improved the salt tolerance of tobacco.

\section{Insect resistance analysis of PeWRKY31-overexpressing transgenic tobacco}

Leaves of WT tobacco and the transgenic lines of similar area and equal quality were placed in the same petri dish to observe the feeding preference of cotton bollworms (Fig. 7a). After feeding for $4 \mathrm{~h}$, it was evident that the insects had focused mainly on WT tobacco leaves, as higher levels of insect chewing damage were visible. After $8 \mathrm{~h}$ of feeding, the area of leaf loss of WT tobacco was nearly $50 \%$, whereas that of all of the transgenic lines was below $10 \%$. These results indicated that PeWRKY31 improved the insect resistance of tobacco.
Leaves of WT tobacco and the transgenic lines of similar area and equal quality were placed in separate petri dishes with cotton bollworms, and the leaf loss was observed after $24 \mathrm{~h}$ (Fig. 7b). The leaf area loss of WT tobacco was greater than that of the transgenic lines, and the leaf losses of the B12 and A12 transgenic lines were less than that of the B5 line, indicating that the B12 and A12 lines had greater insect resistance.

\section{Transcriptome analysis of PeWRKY31-overexpressing transgenic tobacco}

WT tobacco and the B12 transgenic tobacco line overexpressing PeWRKY31 were subjected to transcriptome sequencing. 37,628 and 38,271 expressed genes were detected from the transgenic and WT tobacco lines, respectively, and 1696 unique genes were detected in transgenic tobacco, whereas 2339 unique genes were detected in WT tobacco. The number of differentially expressed genes in transgenic and WT tobacco was 14, 892, with 6978 upregulated genes and 7914 downregulated genes in the transgenic line (Fig. S4-B).

Gene ontology functional classification of genes was used to examine the functional categories of the differentially expressed genes. Among all of the differentially expressed genes, 4753 were annotated to 677 biological processes, 1635 were annotated to 177 cellular components, and 6704 were annotated to 457 molecular functions, among which the differentially expressed genes enriched 44 biological processes, seven cellular components, and 27 molecular functions. The biological processes comprised mainly metabolic processes, the main cellular components were thylakoid and photosystem, and the main molecular functions were catalytic activity and binding (Fig. S4-C).

In addition, the KEGG database was used to analyze the enrichment of the differentially expressed genes in different pathways. 1233 differentially expressed genes of PeWRKY31-overexpressing transgenic tobacco were 

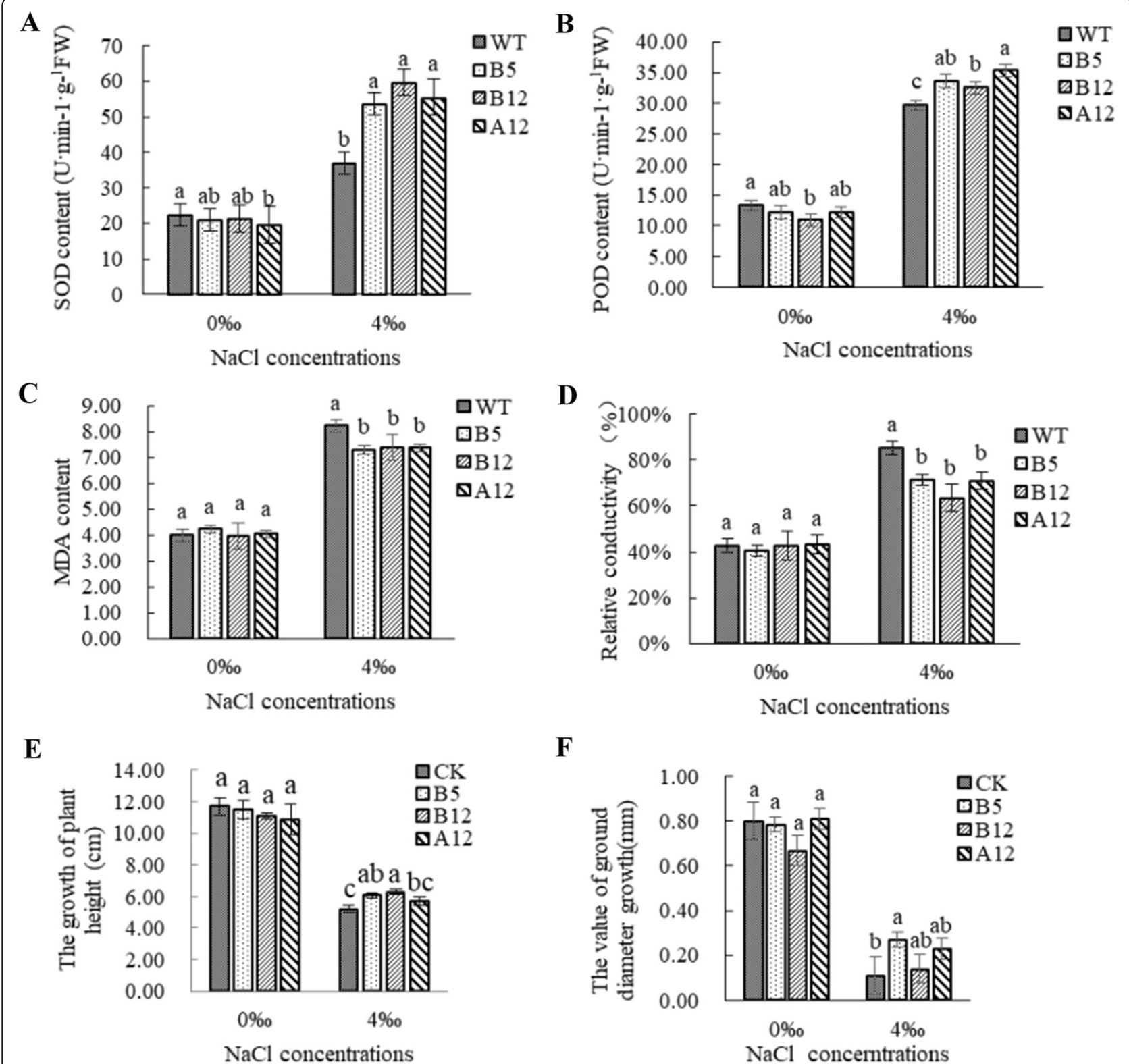

Fig. 6 Effect of salt stress on PeWRKY31-overexpressed tobacco. a: SOD, b: POD, and c: MDA contents under NaCl stress. Variation in the d: relative conductivity, e: plant height, and $\mathbf{f}$ : ground diameter. The means \pm SD of three biological replicates are presented. Error bars represent the standard deviation of the mean. Different letters above the bars indicate a statistically significant difference between the transgenic lines at the $P<0.05$ level according to LSD multiple range test

annotated to 113 specific metabolic pathways. The pathways include those involved in plant hormone signal transduction, plant-pathogen interactions, plant MAPK signaling pathways, carotenoid biosynthesis, terpenoid and ketone biosynthesis, ascorbate metabolism, glutathione metabolism, ubiquinone, and biosynthesis of other terpenoids and quinones. Phenylpropanoid biosynthesis and other pathways listed above are correlated with plant disease, insect resistance, and salt resistance.

In the PeWRKY31-overexpressing transgenic line, the expression of genes related to stress was significantly upregulated. In the glutathione metabolism pathway, the expression of GSH-Px was significantly upregulated. Ascorbate peroxidase (APX), whose gene was also upregulated, scavenges $\mathrm{H}_{2} \mathrm{O}_{2}$ in plants, especially in chloroplasts. In plant hormone signal transduction, the expression of JAR1 was upregulated. The upregulated expression of NPR1 and the binding of TGA transcription factors to the NPR1 promoter can initiate broadspectrum resistance in tobacco. Among the plant MAPK signaling pathways, there were two key pathways related to disease resistance, two related to wounding, and two 


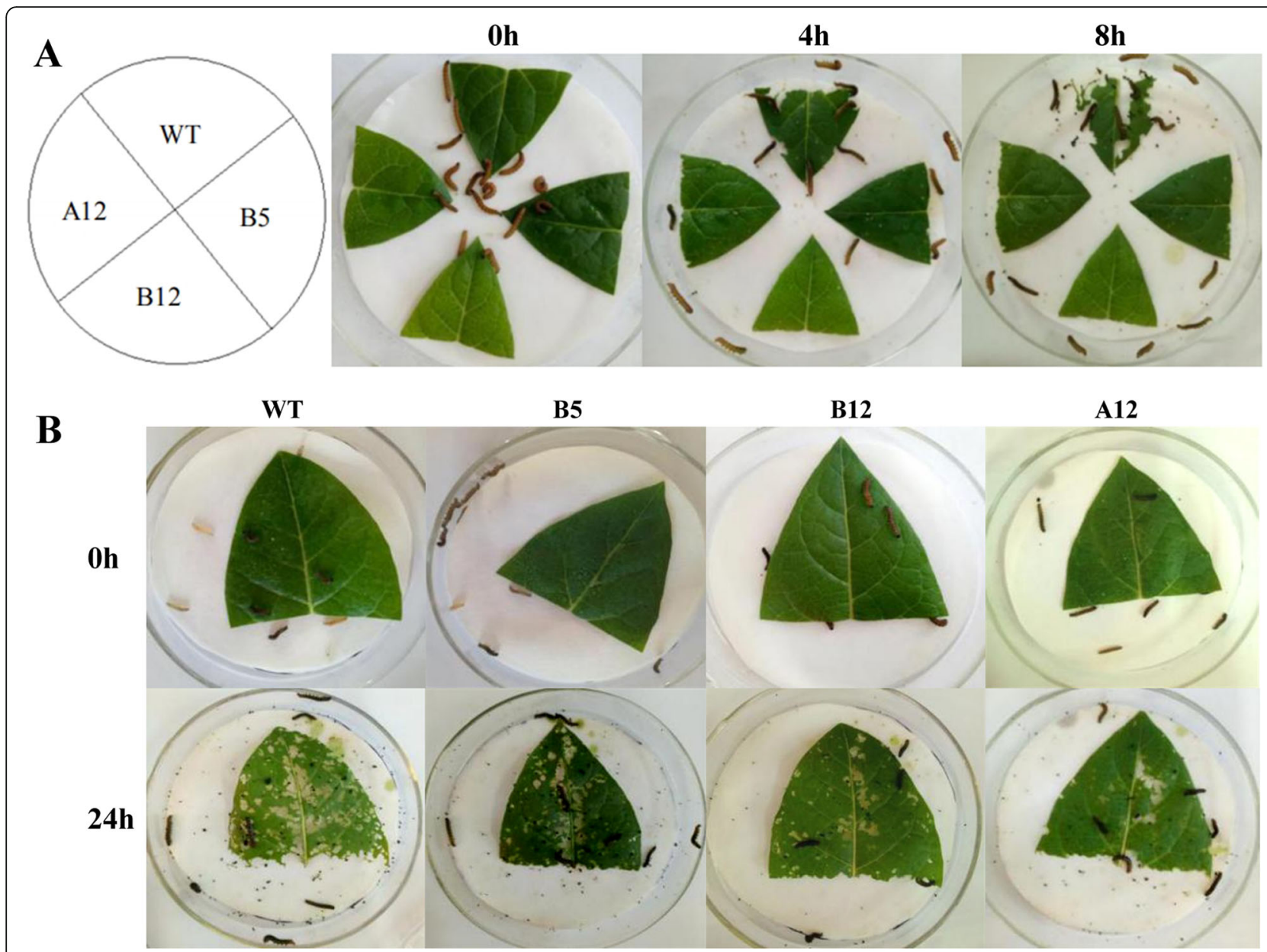

Fig. 7 Feeding experiments of cotton bollworm on PeWRKY31-overexpressing transgenic tobacco. a: Feeding preference (choice or preference). b: Mandatory feeding (no choice)

related to cold resistance, drought resistance, and salinealkali tolerance. In the wounding pathway, the expression of RbohD and OXI1 were upregulated. In plant MAPK signaling pathway, MAPK5, MAPK8, MAPK1, MPK2 and MPK6, which were related to abiotic stress, were upregulated.

To verify the reliability of the differentially expressed genes (DEGs) results, eight upregulated DEGs related to biological and abiotic stress were selected for quantitative reverse transcriptase-polymerase chain reaction (qRT-PCR) verification, with NtActin used as the internal reference gene. Figure 8 shows the sequencing results of the eight genes and the qRT-PCR results. Although the qRT-PCR gene expression results for line B12 were not completely consistent with the sequencing results, the expression patterns of all eight genes used for verification were basically consistent with the DEGs, which showed the accuracy and credibility of the sequencing data. The differences in relative expression of DEGs among different transgenic lines might be caused by different insertion locations, and their expression patterns will also differ by insertion location (see Table S1 for the primer sequences).

\section{Discussion}

Subcellular localization analysis of PeWRKY31

Growing transcriptome databases resulting from continuously developing bioinformatics technologies and high-throughput sequencing projects are increasingly being used for gene mining and functional research [30], and PCR methods are used to obtain the target genes [31]. In this study, qRT-PCR showed that PeWRKY31 responded to biotic and abiotic stresses.

Most activities of WRKY proteins take place in the nucleus. WRKY proteins of Arabidopsis [32] and rice [33] localizes to the nucleus, and fluorescent signals from fusion proteins of PtrWRKY40 in Populus trichocarpa and PtoWRKY60 in Populus tomentosa are observed exclusively in the nucleus [34]. In addition, the OfWRKY3, CaWRKY6 and CaWRKY30 proteins are also located in 


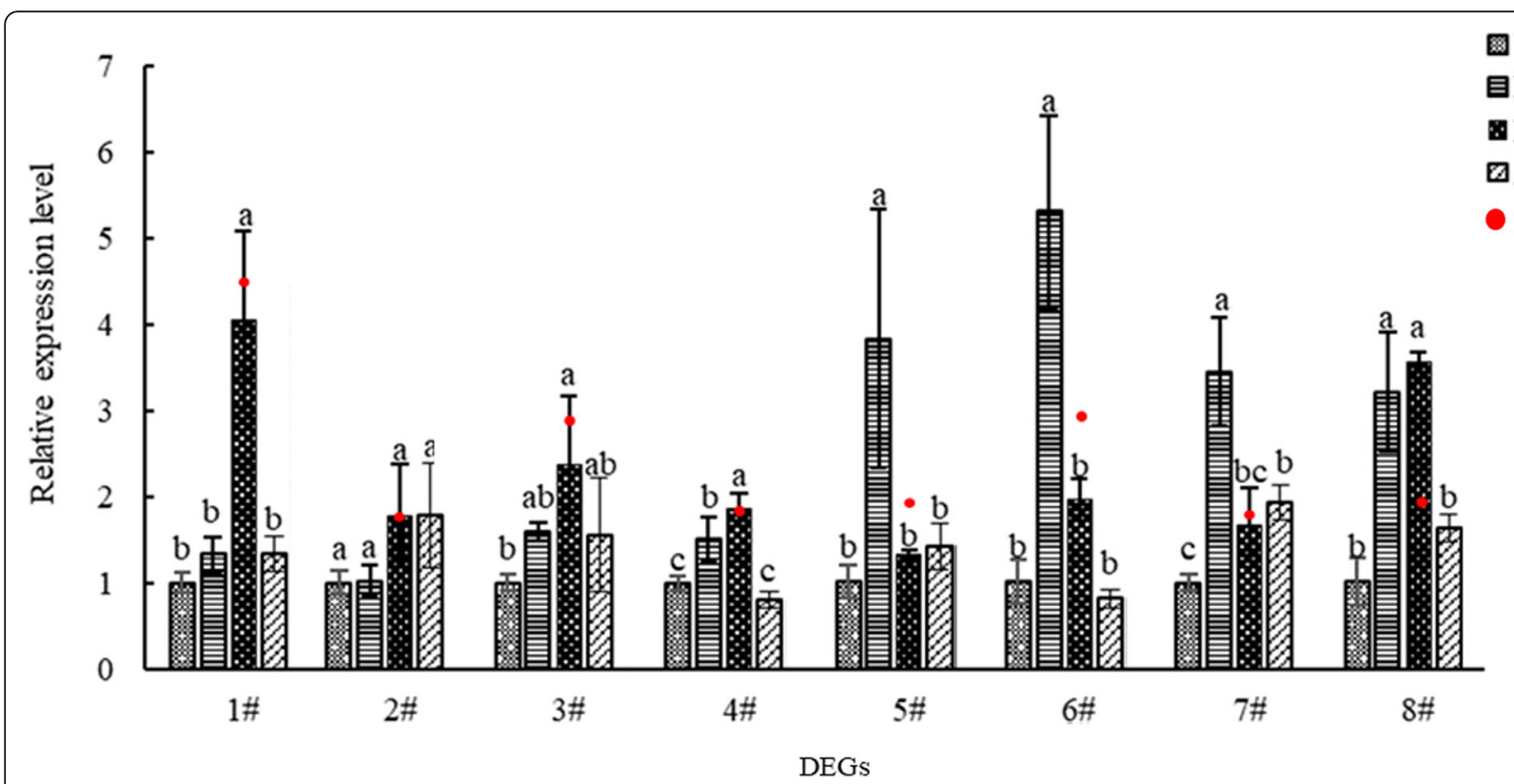

Fig. 8 Relative expression of selected DEGs, compared using qRT-PCR and RNA-Seq. 1\#: LOC107782952; 2\#: LOC107798207; 3\#: LOC107831218; 4\#: LOC107768362; 5\#: LOC107824233; 6\#: LOC107832641; 7\#: LOC107770091; 8\#: LOC107816761; TD: Relative expression level of RNA-seq result. "TD" refers to the ratio of gene expression between the treatment and control groups. After treatmented with difference analysis software shrinkage model, the final logarithm was taken as the base of 2 , namely $\log _{2}$ (Fold Change). The means \pm SD of three biological replicates are presented. Error bars represent the standard deviation of the mean. Different letters above the bars indicate a statistically significant at the $P<0.05$ level according to LSD multiple range test

the nucleus [35]. Fluorescence microscopy reveals that the DAPI staining results of the transient transformation are consistent with the green fluorescent signal, indicating that PeWRKY31 localizes specifically to the nucleus.

\section{Resistance analysis of PeWRKY31-overexpressing tobacco}

In recent years, with increasing number of studies on transcription factors, WRKY transcription factors have been found to be involved in a wide variety of biological processes in plants. Although WRKY genes have been studied in Arabidopsis, rice, and other model plant species $[8,9]$, there have been few studies on WRKY genes in poplar.

Overexpression of genes that respond to salt stress can improve salt tolerance in plants [36-38]. Under salt stress, the contents of antioxidant enzymes increase in chrysanthemum overexpressing DgWRKY malondialdehyde content decreases [39]. Antioxidant enzyme activities in transgenic wheat overexpressing TaWRKY44 were much higher than in WT [22]. The soybean gene GmWRKY12 responded to salt stress, and the MDA content decreased in GmWRKY12-overexpressing soybean [40]. TaWRKY10, TaWRKY44, and TaWRKY19 in wheat responded to salt stress, and improved the tolerance of transgenic plants to salt stress [21, 22, 41]. In this study, a PeWRKY31 overexpression vector was constructed and transformed into tobacco.
Under salt treatment, the SOD and POD activities of PeWRKY31-overexpressing tobacco were significantly higher than those in WT, and the MDA content and electrical conductivity were significantly lower than that in WT. These results align with those of previous studies, suggesting that PeWRKY31 could enhance the salt tolerance of tobacco.

AtWRKY18, AtWRKY40, and AtWRKY60 in Arabidopsis belong to the WRKY IIa + IIb subfamily, and cooperate to regulate ABA-mediated salt stress responses [42, 43]. In Nicotiana attenuate, WRKY3 and WRKY6 respond to chew damage inflicted by herbivorous insects. WRKYs can regulate the transcription level of certain genes by activating signal pathways, such as those involved in salicylic acid (SA), ethylene (ET), and jasmonic acid (JA) [44-46]. Overexpression of AtWRKY18AtWRKY4O or AtWRKY18-AtWRKY60 was found to regulate immunity to insects by regulating the JA and SA pathways in Arabidopsis [23]. AtWRKY28 and AtWRKY75 may enhance the resistance of Arabidopsis to fungal infection through the JA and ET pathways [47]. Overexpression of OsWRKY53 in rice positively controlled resistance to certain insects by activating $\mathrm{H}_{2} \mathrm{O}_{2}$ and inhibiting the synthesis of ethylene [24]. All of these results indicate that WRKYs regulate susceptibility and resistance to insect pests by participating in signal transduction. In this study, Populus $\times$ euramericana 
PeWRKY31 was upregulated after being subjected to biological stress, and overexpression of PeWRKY31 in tobacco increased its resistance to insects. However, additional experiments are needed to determine whether PeWRKY31 functions in Populus in the same manner as in tobacco.

\section{Transcriptome analysis}

In the present study, the transgenic tobacco overexpressing PeWRKY31 showed enhanced responses to biotic and abiotic stresses. Transcriptome KEGG enrichment analysis revealed differentially expressed genes were related to plant hormone signal transduction, plant MAPK signaling pathways, carotenoid biosynthesis, terpenoid and ketone biosynthesis, and other important salttolerance and insect-resistance pathways [44, 45]. Differentially expressed genes were frequent in glutathione metabolism, plant hormone signal transduction, and plant MAPK signaling pathways, all of which responded to biotic and abiotic stresses. GSH-Px catalyzed the conversion of reduced glutathione (GSH) to oxidized glutathione (GSSG), which scavenged harmful free radicals, thus protecting the structure and function of cell membranes against damage by peroxides [48]. Upregulated $A P X$ expression could enhance the ability of plants to tolerate oxidation, which in turn enhanced their ability to resist stress [49]. Upregulated expression of LAR1 could enhance the salt tolerance and insect resistance of plants [48]. Upregulated expression of JAR1 would increase JA-Ile, which promoted the formation of the COI1-JAZ co-receptor complex [50]. This ultimately led to the degradation of the JAZ protein. JAZ degradation can enhance the interaction between $\mathrm{MYC} 2$ and other proteins, activating the expression of the promoter target of MYC2 and enhancing the salt tolerance and insect resistance of tobacco [50]. The upregulated expression of LAR1, $A P X, M Y C$, and MAPK family genes in these pathways likely play an important role in regulating the responses of PeWRKY31-overexpressing transgenic tobacco to diseases, insect pests, and salt stress.

Transgenic tobacco lines differ in their resistance to biotic and abiotic stresses, which may be related to differences in the position of the PeWRKY31 insertion; such differences may also regulate the expression of other DEGs. Downregulated expression of the homologous NtWRKY31 gene in tobacco may be due to the inhibitory effect of overexpression of PeWRKY31. In future research, we will explore the regulatory mechanism of PeWRKY31, and provide a theoretical basis for breeding resistant poplars.

\section{Conclusions}

In this study, PeWRKY31 was isolated from Populus $\times$ euramericana, and its functions were investigated.
Overexpression of PeWRKY31 improved the resistance of transgenic tobacco to salt stress and pest stress. These results not only reveal the roles of WRKY31 in Populus $\times$ euramericana, but also inform the use of WRKYs in future woody plant research. Future projects will include the overexpression of PeWRKY31 in poplar, a model woody plant, to clarify its specific role in responses to biotic and abiotic stresses. These studies will have a goal of the generation of stress-resistant lines, with potentially great economic benefit.

\section{Methods \\ Materials}

The plant materials used were vegetative propagule of Populus $\times$ euramericana and wild-type (WT) tobacco (Nicotiana tabacum L.). The bacterial strains used were Escherichia coli DH10B and Agrobacterium tumefaciens GV3101. The plasmids pUCm-T, pCAMBIA1302, and pBI121 were used for vector construction. The materials were stored in Hebei Key Laboratory of Forest Germplasm Resources and Forest Protection, College of Forestry, Hebei Agricultural University.

\section{Quantitative reverse transcriptase-polymerase chain reaction}

To analyze PeWRKY31 expression in Populus $\times$ euramericana under different stresses and in different overexpressed tobacco lines, RNA was extracted from leaves and reverse-transcribed into cDNA. The purity of the RNA was determined based on the $\mathrm{OD}_{260} / \mathrm{OD}_{280}$ and $\mathrm{OD}_{260} / \mathrm{OD}_{230}$ ratios. A pair of specific primers (Table S1) and SYBR Green Master Mix (Roche) were used for qRT-PCR amplification of PeWRKY31. Eight DEGs were also screened for qRT-PCR based on transcriptomic data (for primer sequences see Table S1). Three independent biological experiments were performed, and actin and $18 \mathrm{~S}$ were used as internal reference. Fold changes in expression levels were calculated by the $\Delta \Delta \mathrm{CT}$ method (fold change $=2-[\Delta \Delta \mathrm{CT}]$ ). Real-time RT-PCR reactions were performed with initial incubation at $95^{\circ} \mathrm{C}$ for $3 \mathrm{~min}$, followed by 40 cycles of $30 \mathrm{~s}$ at $95^{\circ} \mathrm{C}$ and $30 \mathrm{~s}$ at an annealing temperature of $55^{\circ} \mathrm{C}$.

\section{Isolation of PeWRKY31}

Total RNA was extracted from leaves using an RNA extraction kit (Sainuo Biotech, Zhangjiakou, China), and the gel electrophoresis method was used to analyze the quality of the RNA. First-strand cDNA was synthesized by reverse transcription.

Transcriptome data were used to design primers to perform a screen for WRKY genes, resulting in the isolation of the PeWRKY31 cDNA. The program Primer ver. 5.0 was used to design the specific primer set 31\#all F/R based on the cDNA sequence; these primers were used 
for PCR amplification of the target gene from genomic DNA to isolate the full-length PeWRKY31 gene. The PCR cycles used were: an initial $95^{\circ} \mathrm{C}$ for $5 \mathrm{~min}$, followed by 30 cycles of $95^{\circ} \mathrm{C}$ for $30 \mathrm{~s}, 59^{\circ} \mathrm{C}$ for $40 \mathrm{~s}$, and $72^{\circ} \mathrm{C}$ for $2 \mathrm{~min}$. The target fragment was ligated into pUCm$\mathrm{T}$, and then transformed into E. coli $\mathrm{DH} 10 \mathrm{~B}$ competent cells by heat shock. The plasmid inserts of positive clones with ampicillin resistance were sequenced. The clone selected for further study was named as $31 \#$ all+T (Table S1 for primer information).

\section{Analysis of PeWRKY31 sequence}

The coding sequence of PeWRKY31 was inferred using ORFfinder (https://www.ncbi.nlm.nih.gov/orffinder/) at the NCBI web site. The molecular mass and isoelectric point of PeWRKY31 were analyzed with ExPASy (http:// web.expasy.org), and its secondary and tertiary structure were predicted using SOPMA (https://npsa-prabi.ibcp.fr/ cgi-bin/npsa_automat.pl?page=/NPSA/npsa_sopma.html) and SWISSMODEL (https://www.swissmodel.expasy.org/ interactive/DTDUCp/models/), respectively. The sequence was analyzed for potential nuclear localization signals using cNLS Mapper (http://nls-mapper.iab.keio.ac.jp/ cgi-bin/NLS_Mapper_form.cgi), transmembrane regions using TMHMM (http://www.cbs.dtu.dk/services/ TMHMM), signal peptides using seqNLS (http://mleg.cse. sc.edu/seqNLS/), and phosphorylation sites using NetPhos ver. 3.1(http://www.cbs.dtu.dk/services/NetPhos/) [26]. Searches for genes homologous to PeWRKY31 were conducted in the NCBI and Phytozome databases. The predicted amino acid sequences of PeWRKY31 and homologous genes were compared using DNAMAN, and a molecular phylogenetic tree was constructed using the neighbor-joining method in MEGA ver. 7.0.

\section{Construction of GFP fusion subcellular localization vector and overexpression vector}

Using the $31 \#$ all $+\mathrm{T}$ clone as a substrate, the primer sets 31\#subl-orf $\mathrm{F} / \mathrm{R}$ and 31 \#op-orf $\mathrm{F} / \mathrm{R}$ were designed to amplify the predicted PeWRKY31 coding sequence. The expression vectors p31\#op+121and p31\#subl+ 1302 were constructed by double enzyme digestion. The GFP fusion expression vector p31\#subl+1302 and the overexpression vector p31\#op+121 were transformed into competent Agrobacterium tumefaciens GV3101 cells by heat shock. The p31\#subl+ 1302 insert region was amplified using the p31\#orf F/ $\mathrm{R}$ and hpt2jc $F / R$ primer sets to verify the correct conformation of PeWRKY31 and GFP in the vector in Agrobacterium tumefaciens, and likewise the p31\#op+ 121 insert region was amplified with the p31\#orf $F / R$ and 103\# F/R primer sets to verify the correct insertion of PeWRKY31 and nptII.

\section{Subcellular localization analysis of PeWRKY31}

The GFP fusion expression vector and the corresponding vector containing only the GFP gene insert were transiently transformed into WT tobacco leaves by agrobacterium infection. The leaves were observed for fluorescence signal using fluorescence microscopy to determine the subcellular location of GFP: PeWRKY31 fusion protein.

\section{Genetic transformation of tobacco and identification of transgenic lines}

Agrobacterium cells containing the overexpression vector p31\#op+121 were oscillated in liquid LB medium containing kanamycin until reaching an $\mathrm{OD}_{600}$ of $0.4-$ 0.6. Vigorous tobacco vegetative propagule were selected as transformation material. Leaves from the vegetative propagule were cut into sections of approximately $1 \mathrm{~cm}^{2}$, and then immersed in infection solution for about 10 min. The leaf discs were blotted on sterile paper, placed onto MS medium, and cultured in darkness for $36 \mathrm{~h}$. The discs were then transferred to screening medium (MS medium with $2.0 \mathrm{mg} \mathrm{L}^{-1}$ 6-BA, $0.1 \mathrm{mg} \mathrm{L}^{-1}$ IBA, $800 \mathrm{mg} \mathrm{L}^{-1}$ cefatoxime, and $50 \mathrm{mg} \mathrm{L}^{-1}$ kanamycin) for further culture. After 30-50 days of selection, resistant vegetative propagules were transferred to rooting medium (MS medium with $800 \mathrm{mg} \mathrm{L}^{-1}$ cefatoxime and $50 \mathrm{mg} \mathrm{L}^{-1}$ kanamycin) for further rooting screening. Vegetative propagules with roots that had reached $2 \mathrm{~cm}$ in length were transferred to plastic pots containing a 3 : 1:1 mixture of peat: perlite: vermiculite and then grown in greenhouse. After 30 days, the fourth leaf was excised from plants exhibiting sufficient growth, and its DNA was extracted using a CTAB-based method. Two pairs of specific primers, $103 \# \mathrm{~F} / \mathrm{R}$ and $35 \# 35$ sendF with $31 \#-$ JCR, were used for confirmation of transgenic lines by PCR. For PCR controls, a plasmid containing the target gene was used as a positive control $(\mathrm{CK}+)$, and DNA from WT tobacco was used as a negative control (CK-).

\section{Salt stress treatment and physiological index determination of transgenic plants}

For salt stress treatments, WT tobacco and three transgenic lines (B5, B12, A12) were planted in the same plastic pots in soil. The soil was treated with water containing either $0 \%$ or $4 \% \mathrm{NaCl}$, and the plants were grown in greenhouse. Both the treatment and the measurement of the indicators below involved three biological replicates.

After $72 \mathrm{~h}$, specimen leaves of each treatment group were harvested to determine the peroxidase (POD) and superoxide dismutase (SOD) activities, malondialdehyde (MDA) content, and electrical conductivity. POD activity was determined using a method that quantified oxidized guaiacol. SOD activity was determined spectrophotometrically based 
on inhibition of the photochemical reduction of nitroblue tetrazolium. The free MDA content was determined using a thiobarbituric acid method. The relative electrical conductivity was measured using soaking method.

On days 0,5 and 10 of salt stress, the plant height and ground diameter of the three transgenic lines (B5, B12, A12) and the WT were measured, and increases were calculated.

\section{Helicoverpa armigera feeding experiment}

Leaves were excised from the WT and transgenic tobacco lines (B5, B12, A12) grown in soil for 30 days. The leaves of the same weigh were respectively placed in petri dishes with 15 and 8 Helicoverpa armigera (cotton bollworm) and each was repeated three times. The loss rates of leaf ingestion and infestation were recorded for each line.

\section{Transcriptome sequencing}

Leaves of 30-day-old WT and transgenic (line B12) tobacco were sent to Tianjin Nuohe Zhiyuan Biological Company for transcriptome sequencing, and three biological replicates of each sample were used for the RNASeq. The differential gene expression was evaluated and analyzed by GO (http://geneontology.org/). The KEGG (https://www.ncbi.nlm.nih.gov/pmc/articles/PMC102409/) database was used to analyze the pathway enrichment of the differentially expressed genes [45]. The biochemical, metabolic, and signal transduction pathways related to the differentially expressed genes were recorded.

\section{Supplementary Information}

The online version contains supplementary material available at https://doi. org/10.1186/s12870-021-02856-3.

\section{Additional file 1: Table S1. The list of primers.}

Additional file 2: Fig. S1. Predicted structure of PeWRKY31. a: Secondary structure of PeWRKY31. Blue, alpha helix; green, beta turn; red, extended strand; purple, random coil. b: Predicted tertiary structure of PeWRKY31.

Additional file 3: Fig. S2. Prediction of transmembrane structure, signal peptides, and phosphorylation sites on PeWRKY31. a: Prediction of transmembrane structure. b: Prediction of signal peptides. c: Prediction of phosphorylation sites.

Additional file 4: Fig. S3. Structures of plant transformation vectors. a: Subcellular localization vector p31\#subl+1302. b: Overexpression vector p31\#op+121.

Additional file 5: Fig. S4. DEGs and GO functional classification of differential genes in PeWRKY31-overexpressing transgenic and WT tobacco. a: Venn diagram showing the number of DEGs. Yellow represents the DEGs specific to B12, purple represents WT, and orange represents the DEGs common to the two strains. b: Vocano diagram showing the number of DEGs. Red is the up-regulated DEGs, and green is the down-regulated DEGs. c: GO functional classification. BP: Biological process; CC: Cellular component; MF: Molecular function.

Additional file 6.

\section{Abbreviations}

ORF: Open reading frame; TF: Transcription factor; KEGG: Kyoto Encyclopedia of Genes and Genomes; GO: Gene ontology; MAPK: Mitogen-activated protein kinase; GFP: GreenFluorescent Protein; SOD: Superoxide dismutase;
POD: Peroxidase; MDA: Malondialdehyde; WT: Wild type; PCR: Polymerase Chain Reaction

\section{Acknowledgements}

Not application.

\section{Authors' contributions}

$X Y$ and $L Z$ analyzed the data and edited the manuscript. $X Y$ and $Y P$ analyzed the data, and wrote the manuscript. YD and CZ conducted the experiments. $\mathrm{BL}$ conducted the experiments and edited the manuscript. MY and LZ designed the experiments and edited the manuscript. All authors read and approved the manuscript.

\section{Funding}

This study was supported by the National Natural Science Foundation of China (No. 32071790) and the Basic Research Plan Project of Hebei Province (18966801D). These funding bodies had no role in the design of the study, sample collection, analysis or interpretation of data and in writing the manuscript.

\section{Availability of data and materials}

All data and materials are presented in the main paper and additional supporting file. All raw sequence data are available in the National Center for Biotechnology Information (NCBI) website with the SRA accession number of PRJNA694343 (https://www.ncbi.nlm.nih.gov/sra/PRJNA694343).

Ethics approval and consent to participate

There are no ethical issues involved.

Consent for publication

Not applicable.

Competing interests

The authors declare that they have no competing interests.

\section{Author details}

${ }^{1}$ Forest Department, Forestry College, Hebei Agricultural University, Baoding, China. ${ }^{2}$ Hebei Key Laboratory for Tree Genetic Resources and Forest Protection, 071000 Baoding, P. R. China. ${ }^{3}$ Tianjin nuohe medical laboratory co. LTD, Tianjin, China. ${ }^{4}$ College of Landscape Architecture and Tourism, Hebei Agricultural University, Baoding, China. ${ }^{5}$ College of Landscape and Ecological Engineering, Hebei University of Engineering, 056000 Handan, P. R. China.

Received: 16 June 2020 Accepted: 26 January 2021

Published online: 06 February 2021

\section{References}

1. Agarwal M, Hao YJ, Kapoor A, et al. A R2R3 type MYB transcription factor is involved in the cold regulation of CBF genes and in acquired freezing tolerance. J Biol Chem. 2006;281(49):37636-45.

2. Hwang SH, Kwon SI, Jang JY, et al. OsWRKY51, a rice transcription factor, functions as a positive regulator in defense response against Xanthomonas oryzae pv. Oryzae Plant Cell Reports. 2016;35(9):1975-85.

3. Zhang YJ, Wang LJ. The WRKY transcription factor superfamily: its origin cukaryotes and expansion in plants. BMC Evol Biol. 2005;5:1-12.

4. Jiang $Y$, Zeng $B$, Zhao $H N$, et al. Genome-wide transcription factor gene prediction and their expressional tissue-specificities in maize. J Integr Plant Biol. 2012:54(9):616-30.

5. Jin JP, Tian F, Yang DC, et al. PlantTFDB 4.0: toward a central hub for transcription factors and regulatory interactions in plants. Nucleic Acids Res. 2017:45(D1):D1040-5.

6. Mohanta TK, Park YH, Bae H. Novel genomic and evolutionary insight of WRKY transcription factors in plant lineage. Sci Rep. 2016;6(1):1-22.

7. Jiang J, Ma S, Ye N, et al. WRKY transcription factors in plant responses to stresses. J Integr Plant Biol. 2017;59(2):86-101.

8. Li S, Fu Q, Chen L, et al. Arabidopsis thaliana WRKY25, WRKY26, and WRKY33 coordinate induction of plant thermo tolerance. Planta. 2011;233(6):1237-52.

9. Wu X, Shiroto Y, Kishitani S, et al. Enhanced heat and drought tolerance in transgenic rice seedlings overexpressing OsWRKY11 under the control of HSP101 promoter. HSP101 promoter. Plant Cell Rep. 2009;28(1):21-30. 
10. Kim CY, Vo KTX, Nguyen CD, et al. Functional analysis of a cold-responsive rice WRKY gene, OsWRKY71. Plant Biotechnol Rep. 2016;10(1):13-23.

11. Tripathi $P$, Rabara RC, Choudhary MK, et al. The interactome of soybean GmWRKY53 using yeast 2-hybrid library screening to saturation. Plant Signal Behav. 2015;10(7):e1028705.

12. Jia HH, Wang C, Wang F, et al. GhWRKY68 reduces resistance to salt and drought in transgenic Nicotiana benthamiana. PLoS One. 2015;10(3): e0120646.

13. Jiang YQ, Deyholos MK. Functional characterization of Arabidopsis $\mathrm{NaCl}$ inducible WRKY25 and WRKY33 transcription factors in abiotic stresses. Plant Mol Biol. 2009;69(1-2):91-105.

14. Tao Z, Kou Y, Liu H, et al. OsWRKY45 alleles play different roles in abscisic acid signalling and salt stress tolerance but similar roles in drought and cold tolerance in rice. J Exp Bot. 2011;62(14):4863-74

15. Çelik Ö, Meriç S, Ayan A, et al. Epigenetic analysis of WRKY transcription factor genes in salt stressed rice (Oryza sativa L.) plants. Environ Experimental Botany. 2019:159:121-31.

16. Scarpeci TE, Zanor MI, Mueller-Roeber B, et al. Overexpression of AtWRKY3O enhances abiotic stress tolerance during early growth stages in Arabidopsis thaliana. Plant Mol Biol. 2016;83(3):265-77.

17. Wang $\mathrm{H}, \mathrm{Hao} J$, Chen $\mathrm{X}$, et al. Overexpression of rice WRKY89 enhances ultraviolet B tolerance and disease resistance in rice plants. Plant Mol Biol. 2007;65(6):799-815.

18. Gao R, Liu P, Yong Y, et al. Genome-wide transcriptomic analysis reveals correlation between higher WRKY61 expression and reduced symptom severity in Turnip crinkle virus infected Arabidopsis thaliana. Scientific Reports. 2016;6(1):1821-1831.

19. Oh SK, Baek KH, Park JM, et al. Capsicum annuum WRKY protein CaWRKY1 is a negative regulator of pathogen defense. New Phytol. 2008:177(4):977-89.

20. Merz PR, Moser T, Holl J, et al. The transcription factor VWWRKY33 is involved in the regulation of grapevine (Vitis vinifera) defense against the oomycete pathogen Plasmopara viticola. Physiol Plant. 2014;153(3):365-80.

21. Niu CF, Wei W, Zhou QY, et al. Wheat WRKY genes TaWRKY2 and TaWRKY19 regulate abiotic stress tolerance in transgenic Arabidopsis plants. Plant Cell Environ. 2012;35(6):1156-70.

22. Wang $X$, Zeng J, Li Y, et al. Expression of TaWRKY44, a wheat WRKY gene, in transgenic tobacco confers multiple abiotic stress tolerances. Front Plant Sci. 2015;6:615.

23. Xu X, Chen C, Fan B, et al. Physical andfunctional interactions between and WRKY60 transcription factors. Plant Cell. 2006;18(5):1310-26.

24. Hu L, Ye M, Li R, et al. OsWRKY53, a versatile switch in regulating herbivoreinduced defense responses in rice. Plant Signal Behav. 2016;11(4):e1169357.

25. Yu X, Du J, Dong Y, et al. Cloning and salt tolerance analysis of the PnHB7 transcription factor in Populus nigra L. Industrial Crops Products. 2020 https://doi.org/10.1016/j.indcrop.2020.112943.

26. Duan Y, Jiang Y, Ye S, et al. PtrWRKY73, a salicylic acid-inducible popla WRKY transcription factor, is involved in disease resistance in Arabidopsis thaliana. Plant Cell Rep. 2015:34(5):831-41.

27. Wang S, Wang J, Yao W, et al. Expression patterns of WRKY genes in dihaploidPopulus simonii $\times P$. nigrainresponse to osmotic stress and ABA treatment. Plant Growth Regul. 2016;78(3):325-33. https://doi.org/10.1007/ s10725-015-0095-1.

28. Yao J, Shen Z, Zhang Y, et al. Populus euphratica WRKY1 binds the promoter of H+-ATPase gene to enhance gene expression and salt tolerance. J Experimental Botany. 2020;71(4):1527-1539.

29. Li Q, Li H, Niu F, et al. Characterization of AtWRKY72 and identification of its antistress function. J Agric Biotechnol. 2019;27(02):191-203.

30. Zhang Y, Wang XF, Ding ZG, et al. Transcriptome profiling of Gossypium barbadense inoculated with Verticillium dahliae provides a resource for cotton improvement. BMC Genomics. 2013;14(1):637.

31. Ye S, Jiang $Y$, Duan $Y$, et al. Constitutive expression of the poplar WRKY transcription factor PtoWRKY60 enhances resistance to Dothiorella gregaria Sacc. In transgenic plants. Tree Physiol. 2014;34(10):1118-29.

32. Eulgem T, Rushton PJ, Robatzek $S$, et al. The WRKY superfamily of plant transcription factors. Trends Plant Sci. 2000:5(5):199-206.

33. Ross CA, Liu Y, Shen QJ. The WRKY gene family in rice (Oryza sativa). J Integr Plant Biol. 2007:49(6):827-42.

34. Karim A, Jiang Y, Guo L, et al. Isolation and characterization of a subgroup Ila WRKY transcription factor PtrWRKY40 from Populus trichocarpa. Tree Physiol. 2015;35(10):1129-39.
35. Han $Y$, Wu M, Cao L, et al. Characterization of OfWRKY3, a transcription factor that positively regulates the carotenoid cleavage dioxygenase gene OfCCD4 in Osmanthus fragrans. Plant Mol Biol. 2016;91(4-5):485-496.

36. He Y, Li W, Lv J, et al. Ectopic expression of a wheat MYB transcription fector gene, TaMYB73, improves salinity stress tolerance in Arabidopsis thaliana. J Exp Bot. 2011;63(3):1511-22.

37. Liu QL, Xu KD, Pan YZ, et al. Functional analysis of a novel Chrysanthemum WRKY transcription fector gene involved in salt tolerance. Plant Mol Biol Report. 2014;32(1):282-9.

38. Bhaskaran S, Savithramma DL. Co-expression of Pennisetum glaucum vacuolar $\mathrm{Na}+/ \mathrm{H}+$ antiporter and Arabidopsis $\mathrm{H}+$-pyrophosphatase enhances salt tolerance in transgenic tomato. J Exp Bot. 2011;62(15):5561-70.

39. Liang QY, Wu YH, Wang K, et al. Chrysanthemum WRKY gene DgWRKY5 enhances tolerance to salt stress in transgenic Chrysanthemum. Sci Rep. 2017;7(1):4799

40. Shi WY, Du YT, Ma J, et al. The WRKY transcription factor GmWRKY12 confers drought and salt tolerance in soybean. Int J Mol Sci. 2018;19(12):4087.

41. Wang C, Deng PY, Chen LL, et al. A wheat WRKY transcription factor TaWRKY 10 confers tolerance to multiple abiotic stresses in transgenic tobacco. PLoS One. 2013;8(6):e65120.

42. Liu ZQ, Yan L, Wu Z, et al. Cooperation of three WRKY-domain transcription factors WRKY18, WRKY40, and WRKY60 in repressing two ABA-responsive genes ABI4 and ABI5 in Arabidopsis. J Exp Bot. 2012;63(18):6371-92.

43. Chen $\mathrm{H}$, Lai Z, Shi J, et al. Roles of Arabidopsis WRKY18, WRKY40 and WRKY60 transcription factors in plant responses to abscisic acid and abiotic stress. BMC Plant Biol. 2010;10:281.

44. Tholl D. Biosynthesis and biological functions of terpenoids in plants. Adv Biochem Eng Biotechnol. 2015;148:63-106.

45. Zhu JK. Salt and drought stress signal transduction in plants. Annu Rev Plant Biol. 2002;53:247-73.

46. Dong X. SA, JA, ethylene, and disease resistance in plants. Curr Opin Plant Biol. 1998;1(4):316-23.

47. Chen X, Liu J, Lin G, et al. Overexpression of AtWRKY28 and AtWRKY75 in Arabidopsis enhances resistance to oxalic acid and Sclerotinia sclerotiorum. Plant Cell Rep. 2006:32(10):1589-99.

48. Chen P, Zuo L, Yu X, et al. Response mechanism in Populus $\times$ euramericana cv.'74/76' revealed by RNA-seq under salt stress. Acta Physiol Plant. 2018; 40(5):1-11. https://doi.org/10.1007/s11738-018-2676-x.

49. Chen Z, Lu H, Hua S, et al. Cloning and overexpression of the ascorbate peroxidase gene from the yam ( Dioscorea alata ) enhances chilling and flood tolerance in transgenic Arabidopsis. J Plant Res. 2019;132(6):857-66.

50. Wei H, Movahedi A, Xu C, et al. Overexpression of PtDefensin enhances resistance to Septotis populiperda in transgenic poplar. Plant Sci. 2020;292: 110379

\section{Publisher's Note}

Springer Nature remains neutral with regard to jurisdictional claims in published maps and institutional affiliations.
Ready to submit your research? Choose BMC and benefit from:

- fast, convenient online submission

- thorough peer review by experienced researchers in your field

- rapid publication on acceptance

- support for research data, including large and complex data types

- gold Open Access which fosters wider collaboration and increased citations

- maximum visibility for your research: over $100 \mathrm{M}$ website views per year

At BMC, research is always in progress.

Learn more biomedcentral.com/submissions 